\title{
XCDCC: Core Excitation in the Breakup of Exotic Nuclei
}

\author{
N. C. Summers, ${ }^{1}$ F. M. Nunes, ${ }^{1,2, \text { 田 and I. J. Thompson }}{ }^{3}$ \\ ${ }^{1}$ National Superconducting Cyclotron Laboratory, Michigan State University, East Lansing, Michigan 48824, U.S.A. \\ ${ }^{2}$ Department of Physics and Astronomy, Michigan State University, East Lansing, Michigan 48824, U.S.A. \\ ${ }^{3}$ Department of Physics, University of Surrey, Guildford, GU2 5XH, U.K.
}

(Dated: July 7, 2018)

\begin{abstract}
The eXtended Continuum Discretized Coupled Channel (XCDCC) method is developed to treat reactions where core degrees of freedom play a role. The projectile is treated as a multi-configuration coupled channels system generated from a valence particle coupled to a deformed core which is allowed to excite. The coupled channels initial state breaks up into a coupled channels continuum which is discretized into bins, similarly to the original CDCC method. Core collective degrees of freedom are also included in the interaction of the core and the target, so that dynamical effects can occur during the reaction. We present results for the breakup of ${ }^{17} \mathrm{C}={ }^{16} \mathrm{C}+\mathrm{n}$ and ${ }^{11} \mathrm{Be}={ }^{10} \mathrm{Be}+\mathrm{n}$ on ${ }^{9}$ Be. Results show that the total cross section increases with core deformation. More importantly, the relative percentage of the various components of the initial state are modified during the reaction process through dynamical effects. This implies that comparing spectroscopic factors from structure calculations with experimental cross sections requires more detailed reaction models that go beyond the single particle model.
\end{abstract}

PACS numbers: 24.10.Eq, 25.60.Gc, 25.70.De, 27.20.+n

\section{INTRODUCTION}

Over the last decade, technological developments have provided the Radioactive Beam community with detailed data on a large variety of reactions involving nuclei far from stability [1]. Amongst these are breakup reactions, from which properties of loosely bound systems can be extracted [2]. Breakup reactions have been used to determine ground state properties, including binding energies, angular momentum structure, momentum distributions and spectroscopic factors (e.g. 3, 4, 5, 6, 7, 8, 9]), as well as for studying excited state properties and resonant states (e.g. 10]).

Breakup is not only of interest for studying the structure of these nuclei, but also for astrophysical reasons. In a Coulomb dominated process, if we can assume a one step semi-classical approximation [11, it is possible to factorize the Coulomb dissociation cross section of $P+t \rightarrow(c+v)+t$ into a kinematical factor multiplying the photo-dissociation cross section of the projectile $c+v \rightarrow P$, which in turn is easily translated into the capture S-factor at arbitrarily low energies between the fragment and core [12. Many applications of this method have been used in the past 13, 14, 15] and new applications are being developed [16].

In general, we need to improve on the semiclassical models, but still some approximations need to be made, depending on the energy regime, the target, and the specific kinematical conditions of the experimental setup. Recent results show that nuclear contributions extend far out into regions where naively one expects they would be negligible, because of the extended nature of loosely

*Electronic address: nunes@nscl.msu.edu bound wave functions. Coulomb-nuclear interference is also not negligible, the far-field approximation of the Coulomb field is inaccurate, and polarization effects need to be considered (see for example 17, 18, 19, 20, 21, 22]). Even when there are no other bound excited states, the breakup process goes through several continuum states under the influence of the target. These effects have been referred to as dynamical effects or continuum-continuum couplings, and together with other improvements, have helped to solve apparent discrepancies between different experiments 23, 24].

In earlier days, measurements involving dripline nuclei detected only one of the fragments (e.g. 25]). Those inclusive data contained inelastic breakup and transfer (stripping) as well as elastic breakup (diffraction). In the latter, the projectile breaks up and both fragments survive after the process without any excitation of the target. In stripping, one of the fragments in the projectile gets absorbed and/or the target gets excited. The improved beam intensities in most facilities and the new phase of electronics in data acquisition has allowed efficient coupling of several types of detectors, such that, nowadays, data are less integrated and often contain complete kinematics (e.g. [26, 27]). For this reason, theoretical models need to move toward disentangling the various process in their predicted observables. In this work we will focus on the elastic breakup component.

Generally, breakup reactions have been described within a three body model, where the projectile is simplified to a two body system $(P=c+v)$. The relative motion between the core and fragment of the projectile is distinguished from, and then coupled to, the relative motion of the projectile and the target. In the high energy regime, straight-line trajectory or adiabatic approximations are often made [28, 29]. At lower energies, semi-classical methods taking a Rutherford orbit for the 
projectile's trajectory are a possible choice [17, 18, 22]. Over all energy regimes, one of the most successful models has been the Continuum Discretized Coupled Channels method [30]. It includes the scattering states of the projectile completely coupled and is fully quantal. In this model, nuclear and Coulomb are treated on equal footing, thus interference effects appear naturally. Also, any relevant multi-step process within the continuum, or from the ground state to the continuum, are automatically incorporated 21, 31].

As successful as it has been, CDCC [30] has several limitations. One of the most serious is the restriction to projectiles that can be approximated by two bodies in relative motion. The Kyushu group [32 has been expanding the original CDCC method to solve problems where the projectile is of a clear three body nature, such as Borromean systems $\left({ }^{6} \mathrm{He},{ }^{11} \mathrm{Li},{ }^{14} \mathrm{Be}\right.$, etc.). Their method introduces a Gaussian expansion with a complex argument [33], essential for computational efficiency. Results for nuclear and Coulomb breakup have been recently presented [34, 35]. Another limitation is associated with the non relativistic description, and this approximation becomes inaccurate at very high energies. Exploratory work solving the Klein-Gordon equation [36] has shown there to be non-negligible relativistic effects in the Coulomb dissociation of ${ }^{8} \mathrm{~B}$ for experiments at GSI energies. Finally, the projectile-target two-body asymptotics, as an approximation of the pure three body asymptotics, has also been pointed out as a limiting factor in certain reactions [37].

The CDCC method has been applied to many recent examples [23, 31, 38, 39, 40, 41]. For the ${ }^{8} \mathrm{~B}$ breakup measured at Notre Dame where only the ${ }^{7} \mathrm{Be}$ fragment was detected [25], the theoretical predictions agree very well with the data for the kinematic region where only elastic breakup is expected [31]. If the same ${ }^{8} \mathrm{~B}$ structure model is used for the breakup of ${ }^{8} \mathrm{~B}$ at higher energies [42], one needs to artificially adjust the quadrupole strength of the couplings potentials in the reaction [43]. This adjustment cannot be understood by a simple renormalization of the $E 2$ strength in the ${ }^{7} \mathrm{Be}-\mathrm{p}$ continuum [44] and suggests that structure beyond the single particle $p_{3 / 2}$ is required to describe the reaction mechanisms at higher energies. The elastic and inelastic scattering of protons on ${ }^{11} \mathrm{Be}$ in inverse kinematics was examined in the inert core single particle model [45]. They found that serious discrepancies between the theory and data existed in the ${ }^{11} \mathrm{Be}\left\{{ }^{10} \mathrm{Be}\left(0^{+}\right)+\mathrm{n}\right\}$ breakup spectrum in the region around resonances built on a $2^{+}$core.

There are several ways to improve the two body single particle description of the projectile. While the Kyushu group has focused on the description of breakup of a three body projectile, in this work we will discuss a generalization of the CDCC method for a two body projectile where the wave function is no longer single particle. This generalization of the CDCC method, which we denominate eXtended CDCC (XCDCC) 46], includes a coupled channels description of the projectile's initial and final states, but also any dynamical core excitation/deexcitation that may occur in the reaction process.

It is clear that if core excitation/de-excitation happens during the reaction process, this will have implications for not only the excited cross sections, but also for measuring the ground state core distributions. In addition, one expects that the excitation/de-excitation process will depend strongly on the type of reaction, namely high versus low energy, light versus heavy target. Even when there is only a single valence neutron/proton, the valence nucleon may occupy several configurations, in some of which the core is excited. By measuring the gamma rays in coincidence with outgoing fragments, core states can be identified, and spectroscopic factors for the overlap of the projectile ground state with specific core states can be examined [47.

In nuclear dominated knockout reactions on light targets, typically the method of 48] is followed. Therein one calculates the single particle cross section for both diffraction (elastic breakup) and stripping, in an eikonal model, for each ground state configuration of the projectile. The single particle cross sections for each state are then multiplied by the spectroscopic factor for that configuration, calculated within a shell model, and the total knockout cross sections is obtained by adding the single particle contributions incoherently. Improvements on the eikonal approximation for the breakup contribution [49, 50] have been examined using CDCC. These models, however, are still single particle and neglect any interference between configurations, as well as any dynamical excitation of the core in the reaction. We will examine the importance of these effects in this paper.

In Coulomb dissociation, by contrast, a more simplistic approach is typically assumed: that the relative probabilities do not change during the reaction, and the contribution from excited core components is negligible [9]. Thus the ratio of the theoretical cross section, for the groundstate-core configuration, to the experimental cross section is often taken to be directly the spectroscopic factor for the ground state.

Contributions from excited core components in Coulomb dissociation have previously been estimated [51], by calculating the contribution from excited core components separately, and adding them incoherently, following the method of [48]. The Coulomb dissociation was calculated using DWBA and Adiabatic reaction models. In [51], it was concluded that the Coulomb dissociation to excited core states was very small, only a few percent, very much less than in nuclear breakup. They then suggest that since the cross section from excited core states has a negligible contribution to the total Coulomb breakup cross section, one could compare the single particle theoretical cross section to the experimental cross section to obtain the spectroscopic factor for the core in its ground state. One has to consider the many approximations assumed in the theory. This model neglected any interference between the configurations and any dynamical (de-)excitation of the core. These effects 
can be examined with XCDCC, but Coulomb dominated reactions will be a subject of a future publication.

One simple way to introduce collective degrees of freedom to the core is to assume it behaves as a perfect rotor. Rotor+nucleon coupled channels models for loosely bound systems have been applied to a number of light nuclei on the dripline (e.g. 52, 53, 54]). This type of structure model for the projectile has been also used in the calculations of reaction observables 55,56 .

In 55., the coupled wave functions are used to compute the $\mathrm{B}(\mathrm{E} 1)$ electric excitation function from the ground state into the continuum. The interference between the states with the same core spin in a pure Coulomb dissociation process is then studied within the semi-classical first order approximation [55]. Interference effects between states with different core spins were neglected since this model does not include inelastic transitions caused by the target. These interference effects were shown to be important in ${ }^{17} \mathrm{C}$ on ${ }^{208} \mathrm{~Pb}$ at $67 \mathrm{~A} \mathrm{MeV}$.

In [56], the eikonal approximation is used to calculate the total cross sections for the breakup of a deformed core plus nucleon system. Results for ${ }^{17} \mathrm{C}$ breakup on ${ }^{9} \mathrm{Be}$ at $62 \mathrm{~A} \mathrm{MeV}$ show a dramatic increase of the cross section with the quadrupole deformation parameter. Contrary to the semi-classical first order approximation, this model includes dynamical effects, but only total cross sections are obtained. It can be interpreted as a precursor to the new model presented here.

There is a wide variety of problems that can be investigated with XCDCC, and this is the first chapter where the formalism is given in detail and integrated observables for two cases are presented, namely ${ }^{17} \mathrm{C}$ and ${ }^{11} \mathrm{Be}$ breakup on ${ }^{9} \mathrm{Be}$ at $\approx 60 \mathrm{~A} \mathrm{MeV}$, both measured at MSU [6, 7]. We present a comparison with the data of [7] in a shorter paper [46].

In section II the theoretical development is presented. A description of the structure models used for the projectiles is detailed in section [III Numerical details for the examples considered are given in section IV Following, in section $\nabla$ the results are detailed and discussed. Finally, section VI contains the conclusions drawn from our study and an outlook for future work.

\section{THEORETICAL DEVELOPMENTS}

The breakup of a projectile $P$ consisting of a valence particle $(v)$ loosely bound to a core $(c)$ on a target $t$ can be modelled as a three body scattering problem. The three body Hamiltonian for the system has the form:

$$
H=T_{\boldsymbol{R}}+H_{\text {proj }}+V_{c t}+V_{v t}
$$

where $T_{\boldsymbol{R}}$ represents the kinetic energy operator for the projectile-target system, $H_{\text {proj }}$ is the internal Hamiltonian of the projectile and $U_{c t}\left(U_{v t}\right)$ is the interaction between the core (valence) particle and the target. The Hamiltonian is usually written in the Jacobi coordinates $(\boldsymbol{R}, \boldsymbol{r})$ where $\boldsymbol{R}$ is the coordinate for the center of mass of

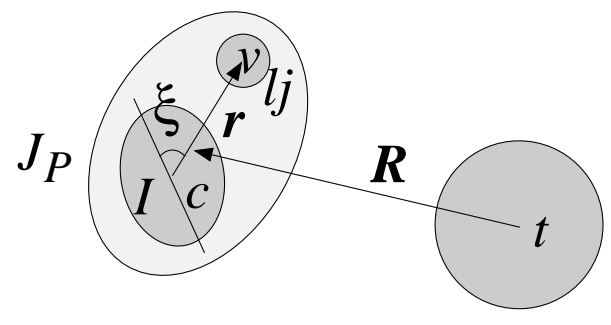

FIG. 1: Coordinates for the three body scattering problem to be considered.

the projectile relative to the center of mass of the target, and $\boldsymbol{r}$ is the coordinate of the valence particle relative to the center of mass of the core as shown in Fig. 1.

The full three body wave function for the system with definite total spin $J_{T}$ is expanded over the states of the projectile, both bound and unbound:

$$
\begin{aligned}
& \Psi_{J_{T}}^{M_{T}}(\boldsymbol{R}, \boldsymbol{r}, \xi) \\
& =\sum_{\alpha} \Psi_{\alpha}^{J_{T}}(R)\left[\left[Y_{L}(\hat{\boldsymbol{R}}) \otimes \Phi_{J_{P}}^{i n}(\boldsymbol{r}, \xi)\right]_{J} \otimes \Phi_{J_{t}}\left(\xi_{t}\right)\right]_{J_{T}}^{M_{T}}
\end{aligned}
$$

The XCDCC states, labelled by $\alpha \equiv\left\{L, J_{P}, J, J_{t}, i, n\right\}$, describe the projectile state $\left\{J_{P}, i, n\right\}$, where $J_{P}$ is the total spin of the projectile, $i$ is the discretization in relative momentum and $n$ is an additional boundary condition that is required to describe coupled channels bins and is discussed later. In this paper we assume the target is structureless, therefore removing the dependence on $\left(\xi_{t}\right)$ in Eq. (2). The projectile-target orbital angular momentum, $L$, and the spin of the target $J_{t}$ all couple to a total spin of the three-body system $J_{T}$. We use the notation

$$
\left\langle\hat{\boldsymbol{R}}, \boldsymbol{r}, \xi \mid \alpha ; J_{T}\right\rangle=\left[\left[Y_{L}(\hat{\boldsymbol{R}}) \otimes \Phi_{J_{P}}^{i n}(\boldsymbol{r}, \xi)\right]_{J} \otimes \Phi_{J_{t}}\right]_{J_{T}}
$$

to describe the angular momentum and projectile wave function compactly.

If the interaction between the fragments in the projectile depends only on $\boldsymbol{r}$, the projectile Hamiltonian $H_{\text {proj }}$ generates single particle bound state wave functions, and single particle continuum states which can later be discretized into continuum bins. In this work, we wish to include an additional degree of freedom, $\xi$, concerning the projectile's core, and allow the core states to be coupled together. For simplicity we take a rotational model for the core [52], but the evaluation of the coupled channels bins and potential matrix elements are not specific to the choice of this collective model. We consider that the core-valence interaction depends on core collective degrees of freedom $\xi$ :

$$
H_{\text {proj }}=T_{\boldsymbol{r}}+V_{v c}(\boldsymbol{r}, \xi)+h_{\text {core }}(\xi),
$$

where $T_{\boldsymbol{r}}$ is the core-valence kinetic energy operator, and $h_{\text {core }}(\xi)$ is the intrinsic Hamiltonian for the core. We define $\varphi_{I}$ to be the eigen function of the core corresponding 
to eigen-energies $\varepsilon_{I}$. Now the projectile ground state contains contributions from different core states, in a fully coupled manner:

$$
\Phi_{0}(\boldsymbol{r}, \xi)=\sum_{a} \phi_{a}(r)\left[\left[Y_{l}(\hat{\boldsymbol{r}}) \otimes \chi_{s}\right]_{j} \otimes \varphi_{I}(\xi)\right]_{J_{P}}
$$

We use the subscript 0 on the projectile wave function to denote the ground state (the supercripts $(i, n)$ in Eq. (3) are superfluous for bound states). Here, a given channel $a$ depends on a set of quantum numbers describing the relative $(l)$ and total $(j)$ angular momentum of the valence particle, with fixed spin $s$, which couples to the core spin $I$. We label each channel which can couple to total spin $J_{P}$ by $a \equiv\{l, j, I\}$. We use the notation

$$
\left\langle\hat{\boldsymbol{r}}, \xi \mid(l s) j, I ; J_{P}\right\rangle=\left[\left[Y_{l}(\hat{\boldsymbol{r}}) \otimes \chi_{s}\right]_{j} \otimes \varphi_{I}(\xi)\right]_{J_{P}}
$$

to describe the angular momentum and core wave function compactly. Previous work [52] gives details of such rotor $+\mathrm{n}$ models.

In addition to the dependence of the internal Hamiltonian of the projectile on $\xi$, the core-target interaction $V_{c t}(\boldsymbol{R}, \xi)$ also contains the dependence on the degrees of freedom of the core and therefore, for a breakup process within this model, the core-target interaction can excite/de-excite the core during the reaction process. The coupling matrix elements of this deformed potential will be discussed in section $\Pi$ B.

\section{A. Construction of projectile coupled channels bins}

The projectile's $(c+v)$ scattering states for a given incoming kinetic energy $E_{k}$ are solutions of a coupled channels equation which can be reduced to the following radial form:

$$
\left[E_{k}-\varepsilon_{a}+\varepsilon_{n}-T_{a}\right] f_{a: n}\left(r ; k_{a n}\right)=\sum_{a^{\prime}} V_{a: a^{\prime}}(r) f_{a^{\prime}: n}\left(r ; k_{a^{\prime} n}\right)
$$

where $a$ and $n$ stand for outgoing and incoming boundary conditions respectively, $E_{k}=\hbar^{2} k^{2} / 2 \mu_{v c}$ is the corevalence relative energy, $k$ is the core-valence relative momentum, and the reduced mass is $\mu_{v c}$. The momentum that appears in the scattering wave, $k_{a n}$, is the asymptotic momentum available in particular channel an, and is defined by

$$
k_{a n}^{2}=k^{2}-\frac{2 \mu_{v c}}{\hbar^{2}}\left[\varepsilon_{a}-\varepsilon_{n}\right],
$$

where $\varepsilon_{a}$ is the energy of the core in the outgoing channel $a$, and $\varepsilon_{n}$ is the energy of the core in the incoming channel, $n$. The kinetic energy operator,

$$
T_{a}=-\frac{\hbar^{2}}{2 \mu_{v c}}\left[\frac{\mathrm{d}^{2}}{\mathrm{~d} r^{2}}-\frac{l(l+1)}{r^{2}}\right],
$$

is defined for the angular momentum $l$ of the core-valence system in channel $a$. The coupling potential matrix is

$$
V_{a: a^{\prime}}(r)=\left\langle(l s) j, I ; J_{P}\left|V_{v c}(\boldsymbol{r}, \xi)\right|\left(l^{\prime} s\right) j^{\prime}, I^{\prime} ; J_{P}\right\rangle,
$$

where $a \equiv\{l, j, I\}$ are the outgoing channel quantum numbers.

The open scattering wave functions are obtained by matching to the asymptotic boundary condition,

$$
f_{a: n}(r ; k)=\frac{\mathrm{i}}{2}\left[H_{n}^{-}(k r) \delta_{a n}-H_{a}^{+}(k r) S_{a: n}^{J_{P}}\right],
$$

at $r_{\text {match }}$ to obtain the coupled channels square $S$-matrix, $S_{a: n}^{J_{P}}$. Here $H_{a}^{( \pm)}(k r)$ are the diagonal Coulomb functions. Inelastic channels with excited core components will remain closed unless the relative energy is higher than the core energy or the incoming channel has an excited core component. If the available energy for channel $(a n)$ is negative, then the wave function $f_{a: n}\left(r ; k_{a n}\right)$ will be closed with separation energy $\hbar^{2} k_{a n}^{2} / 2 \mu_{v c}$.

In principle, coupled channels scattering wave functions could be used directly to calculate the one-step breakup process. However, as multi-step effects can be extremely important, we opt to discretize the coupled channels set, into square integrable bin wave functions. The generalization of the standard binning procedure 30 to coupled channels bins is described below.

For each projectile $J_{P}$ state in the continuum, we define coupled channels bin wave functions that are square integrable, resulting from a superposition of pure scattering states (calculated from Eq. (7)) within a momentum bin $\left[k_{i-1}, k_{i}\right]$. Each momentum interval is labelled by $i$. The coupled channels bin wave function are written as

$$
u_{a: n}^{i}(r)=\sqrt{\frac{2}{\pi N_{n}^{i}}} \int_{k_{i-1}}^{k_{i}} \mathrm{~d} k g_{n}(k) f_{a: n}\left(r ; k_{a n}\right)
$$

where, as before, $k$ is the relative momentum between core and valence fragments. Here, $k_{0}$ represents the breakup threshold between the core and valence particles.

The weight factor of the coupled channels bins, $g_{n}(k)$, is chosen to be $\mathrm{e}^{-\mathrm{i} \delta_{n}(k)}$, where $\delta_{n}(k)$ is the phase shift of channel $a=n$ (i.e. the phase obtained from the diagonal of the $S$-matrix). In the one channel case, this phase makes the wave functions real 21]. The coupled channel bins are now complex, and the full complex wave function is used in the CDCC equations up to the matching radius $r_{\text {match }}$. By choosing the weight factor as the phase of channel $a=n$, the wave functions are approximately real. This is useful when matching to the asymptotic Coulomb functions 57] in the CDCC equations, as the long-range Coulomb functions become approximately real.

The normalization factor is

$$
N_{n}^{i}=\int_{k_{i}-1}^{k_{i}}\left|g_{n}(k)\right|^{2} \mathrm{~d} k
$$

Since our weighting has $\left|g_{n}(k)\right|^{2}=1$, the normalization is simply $N_{n}^{i}=k_{i}-k_{i-1}$, and the average energy of bin $\alpha$ is $\hat{\varepsilon}_{\alpha}=\hbar^{2}\left(k_{i}^{3}-k_{i-1}^{3}\right) / 6 \mu_{v c}\left(k_{i}-k_{i-1}\right)$ [31].

The full bin wave function describing the projectile state $J_{P}$, for momentum bin $i$ and incoming wave $n$, 
is the sum of the coupled channels bins obtained from Eq. (12):

$$
\Phi_{J_{P}}^{i n}(\boldsymbol{r}, \xi)=\sum_{a} u_{a: n}^{i}(r)\left\langle\hat{\boldsymbol{r}}, \xi \mid(l s) j, I ; J_{P}\right\rangle .
$$

The incoming channels form an orthogonal set and therefore projectile wave functions carry the label $n$. It is important to note that since the bin wave functions appear in the bra in the three-body $T$-matrix, these incoming wave boundary conditions on the scattering wave relate to the outgoing channel of the breakup fragments.

\section{B. The three body coupling potentials}

The scattering problem for the three body Hamiltonian, Eq. (1) $H \Psi=E \Psi$, can be reduced to a set of radial coupled channels equations in $R$ when using the expansion Eq. (2). Then the scattering coupled channels equation becomes

$$
\left[E_{\alpha}-T_{L}-U_{\alpha: \alpha}^{J_{T}}(R)\right] \Psi_{\alpha}^{J_{T}}(R)=\sum_{\alpha^{\prime} \neq \alpha} U_{\alpha: \alpha^{\prime}}^{J_{T}}(R) \Psi_{\alpha^{\prime}}^{J_{T}}(R)
$$

where the kinetic energy operator is

$$
T_{L}=-\frac{\hbar^{2}}{2 \mu_{P t}}\left[\frac{\mathrm{d}^{2}}{\mathrm{~d} R^{2}}-\frac{L(L+1)}{R^{2}}\right],
$$

and $\mu_{P t}$ is the reduced mass of the projectile-target system. The energy of each channel $E_{\alpha}=E-\hat{\varepsilon}_{\alpha}$ is the total energy of the three-body system minus the average energy of bin $\alpha$. The incident center-of-mass energy will be $E_{0}$, as $\alpha=0$ represents the ground state of the projectile.

The coupling potentials for the elastic breakup of the projectile (the process where the target remains in its ground state) are

$$
U_{\alpha: \alpha^{\prime}}^{J_{T}}(R)=\left\langle\alpha ; J_{T}\left|V_{c t}(\boldsymbol{R}, \boldsymbol{r}, \xi)+V_{v t}(\boldsymbol{R}, \boldsymbol{r})\right| \alpha^{\prime} ; J_{T}\right\rangle,
$$

where $\alpha$ describes the angular momentum as defined in Eq. (3). The projectile-target interaction potential is the sum of the core-target $\left(V_{c t}\right)$ and valence-target $\left(V_{v t}\right)$ potentials, and contains both nuclear and Coulomb terms. The valence-target interaction can be treated as before
[58], by the theory of Appendix [B The new physical element is in the core-target interaction, which now depends on the core degrees of freedom $(\xi)$.

The core-target interaction can be expanded in multipoles of $Q$,

$$
V_{c t}(\boldsymbol{R}, \boldsymbol{r}, \xi)=\sum_{Q} \hat{Q}^{2} V_{c t}^{Q}\left(\boldsymbol{r}_{\boldsymbol{c}}\right) \sum_{q} C_{Q q}\left(\hat{\boldsymbol{r}_{\boldsymbol{c}}}\right) C_{Q q}^{*}(\xi)
$$

where $\boldsymbol{r}_{\boldsymbol{c}}=\boldsymbol{R}-\gamma \boldsymbol{r}$ is the core-target coordinate, with $\gamma=$ $m_{v} / m_{P}$ as the ratio of the valence mass to the projectile mass, and $C_{Q q}\left(\hat{\boldsymbol{r}_{\boldsymbol{c}}}\right)$ is related to the spherical harmonic,

$$
Y_{Q q}\left(\hat{\boldsymbol{r}_{\boldsymbol{c}}}\right)=\hat{Q} C_{Q q}\left(\hat{\boldsymbol{r}_{\boldsymbol{c}}}\right) / \sqrt{4 \pi},
$$

where we use the notation $\hat{Q}=\sqrt{2 Q+1}$.

Next, the product $r_{c}^{Q} C_{Q q}\left(\hat{\boldsymbol{r}_{\boldsymbol{c}}}\right)$ can be expanded in multipoles $\lambda$ for the $\boldsymbol{r}$ and $\boldsymbol{R}$ coordinates, as in [59]:

$$
\begin{aligned}
r_{c}^{Q} C_{Q q}\left(\hat{\boldsymbol{r}_{\boldsymbol{c}}}\right)= & \hat{Q}^{3} \sum_{\lambda \mu} \sqrt{\frac{(2 Q) !}{(2 \lambda) ![2(Q-\lambda)] !}} R^{\lambda}(-\gamma r)^{Q-\lambda} \\
& \times C_{\lambda \mu}(\hat{\boldsymbol{R}}) C_{Q-\lambda, q-\mu}(\hat{\boldsymbol{r}})\left(\begin{array}{ccc}
Q-\lambda & \lambda & Q \\
q-\mu & \mu & -q
\end{array}\right) .
\end{aligned}
$$

The multipole $Q$ for the core-target potential $V_{c t}^{Q}\left(\boldsymbol{r}_{\boldsymbol{c}}\right)$ can be expanded in multipoles of $K$,

$$
\frac{V_{c t}^{Q}\left(\boldsymbol{r}_{\boldsymbol{c}}\right)}{r_{c}^{Q}}=\sum_{K} \hat{K}^{2} V_{c t}^{Q K}(r, R) \sum_{k} C_{K k}(\hat{\boldsymbol{R}}) C_{K k}^{*}(\hat{\boldsymbol{r}})
$$

where the radial potential dependence is given by

$$
V_{c t}^{Q K}(r, R)=\frac{1}{2} \int_{-1}^{+1} \frac{V_{c t}^{Q}\left(\boldsymbol{r}_{\boldsymbol{c}}\right)}{r_{c}^{Q}} P_{K}(u) \mathrm{d} u \quad ; \quad u=\hat{\boldsymbol{R}} \cdot \hat{\boldsymbol{r}}
$$

Combining Eqs. (18), (20) and (21), we find that the spherical harmonics in $\boldsymbol{R}$ can be coupled to a new multipole $\Lambda$ and, similarly, the spherical harmonics in $\boldsymbol{r}$ can be coupled to another multipole $\Lambda^{\prime}$. The angular momentum structure gives three spherical harmonics in $\hat{\boldsymbol{R}}, \hat{\boldsymbol{r}}, \xi$ coupling to form a tripolar spherical harmonic of rank zero. Summing over the angular momentum projections $(q, \mu, k)$, the core-target potential operator can then be reduced (according to Appendix to

$$
\begin{aligned}
V_{c t}(\boldsymbol{R}, \boldsymbol{r}, \xi)= & \sum_{K Q \lambda} V_{c t}^{Q K}(r, R) R^{\lambda}(-\gamma r)^{Q-\lambda} \sqrt{\frac{(2 Q) !}{(2 \lambda) ![2(Q-\lambda)] !}}(-1)^{Q} \hat{Q} \hat{K}^{2} \\
& \times \sum_{\Lambda \Lambda^{\prime}} \hat{\Lambda} \hat{\Lambda}^{\prime 2}\left(\begin{array}{ccc}
K & \lambda & \Lambda \\
0 & 0 & 0
\end{array}\right)\left(\begin{array}{ccc}
K & Q-\lambda & \Lambda^{\prime} \\
0 & 0 & 0
\end{array}\right)\left\{\begin{array}{ccc}
\Lambda^{\prime} & \Lambda & Q \\
\lambda & Q-\lambda & K
\end{array}\right\}\left(C_{\Lambda}(\hat{\boldsymbol{R}}) \cdot\left\{C_{\Lambda^{\prime}}(\hat{\boldsymbol{r}}) \otimes C_{Q}(\xi)\right\}_{\Lambda}\right) .
\end{aligned}
$$


The transition couplings of Eq. (17) are calculated as matrix elements of Eq. (23), assuming the coupling order defined in Eq. (2). It is useful to write the transition couplings in terms of a transition potential which only depends on the overall projectile initial and final states, $J_{P}^{\prime}, i^{\prime}, n^{\prime}$ and $J_{P}, i, n$, and the transition multipolarity $\Lambda$. Combining the potential operator from Eq. (23) and the matrix elements from Appendix $\mathrm{A}$ we write the coretarget three-body couplings as

$$
\begin{aligned}
U_{\alpha: \alpha^{\prime}}^{J_{T}}(R)= & \hat{L} \hat{L}^{\prime} \hat{J_{P}} \hat{J}_{P}^{\prime}(-1)^{J_{P}+J} \sum_{\Lambda}(-1)^{\Lambda} \hat{\Lambda}^{2} \\
& \times\left(\begin{array}{ccc}
\Lambda & L & L^{\prime} \\
0 & 0 & 0
\end{array}\right)\left\{\begin{array}{ccc}
J_{P} & J_{P}^{\prime} & \Lambda \\
L^{\prime} & L & J
\end{array}\right\} F_{J_{P} i n: J_{P}^{\prime} i^{\prime} n^{\prime}}^{\Lambda}(R) .
\end{aligned}
$$

The form factor is the sum over $K Q \lambda$ multipoles and the projectile coupled channels ( $a$ and $\left.a^{\prime}\right)$,

$$
F_{J_{P} i n: J_{P}^{\prime} i^{\prime} n^{\prime}}^{\Lambda}(R)=\sum_{\substack{K Q \lambda \\ a a^{\prime}}} R_{a i n: a^{\prime} i^{\prime} n^{\prime}}^{K Q \lambda}(R) P_{a: a^{\prime}}^{K Q \lambda: \Lambda}
$$

where the radial dependence is defined by the integral:

$$
\begin{aligned}
& R_{a i n: a^{\prime} i^{\prime} n^{\prime}}^{K Q \lambda}(R) \\
& =\hat{K} \int_{0}^{R_{m}} u_{a: n}^{i}(r)^{*} V_{c t}^{Q K}(r, R) R^{\lambda}(-\gamma r)^{Q-\lambda} u_{a^{\prime}: n^{\prime}}^{i^{\prime}}(r) \mathrm{d} r .
\end{aligned}
$$

The remaining angular momentum dependence is summarized as

$$
\begin{aligned}
P_{a: a^{\prime}}^{K Q \lambda: \Lambda}= & (-1)^{j^{\prime}+l+l^{\prime}+s+Q} \hat{Q} \hat{K} \hat{j} \hat{j}^{\prime} \hat{l^{\prime}} \sqrt{\frac{(2 Q) !}{(2 \lambda) ![2(Q-\lambda)] !}}\left(\begin{array}{ccc}
K & \lambda & \Lambda \\
0 & 0 & 0
\end{array}\right)\left\langle I\left\|C_{Q}(\xi)\right\| I^{\prime}\right\rangle \\
& \times \sum_{\Lambda^{\prime}}{\hat{\Lambda^{\prime}}}^{2}\left(\begin{array}{ccc}
K & Q-\lambda & \Lambda^{\prime} \\
0 & 0 & 0
\end{array}\right)\left(\begin{array}{ccc}
\Lambda^{\prime} & l & l^{\prime} \\
0 & 0 & 0
\end{array}\right)\left\{\begin{array}{ccc}
\Lambda^{\prime} & \Lambda & Q \\
\lambda & Q-\lambda & K
\end{array}\right\}\left\{\begin{array}{ccc}
j & j^{\prime} & \Lambda^{\prime} \\
l^{\prime} & l & s
\end{array}\right\}\left\{\begin{array}{ccc}
J_{P} & J_{P}^{\prime} & \Lambda \\
j & j^{\prime} & \Lambda^{\prime} \\
I & I^{\prime} & Q
\end{array}\right\} .
\end{aligned}
$$

The angular momentum coupling in Eq. (27) contains the matrix element $\left\langle I\left\|C_{Q}(\xi)\right\| I^{\prime}\right\rangle$, which describes elastic and inelastic excitations of the core through its scattering by the target. In this paper we use a rotational model for the core, for which this matrix element has a simple solution (Appendix C]).

This matrix element $\left\langle I\left\|C_{Q}(\xi)\right\| I^{\prime}\right\rangle$ is left separate in Eq. (27) since, in general, other models could be used to induce the inelastic scattering of the core. Given a coretarget interaction with core degrees of freedom, $\xi$, and multipole expansion, $Q$, the inelastic modes can be calculated, and along with radial coupling potentials [Eq. (10)] for the core-valence interaction, XCDCC opens the door for other models of the core which go beyond the collective model.

The monopole couplings for the core-target interaction $[Q=0$ in Eqs. [2427)] are evaluated in Appendix B We see by comparing Eq. (24) and Eq. (B1) that including extra core degrees of freedom for the core does not increase the complexity of the final form factors, since they both depend only on a multipolarity and the transition $J_{P}^{\prime} \rightarrow J_{P}$. Thus the form factors can be pre-calculated efficiently. To reduce memory requirements, the individual components $R_{a i n: a^{\prime} i^{\prime} n^{\prime}}^{K Q \lambda}(R) P_{a: a^{\prime}}^{K Q \lambda: \Lambda}$ can be summed so only the final $F_{J_{P} i n: J_{P}^{\prime} i^{\prime} n^{\prime}}^{\Lambda}(R)$ stored. A new version of FRESCO [58] was created for this purpose.

The only increase in the size of the calculation compared with the single particle calculations, with no core degrees of freedom, is the increase in the model space due to the extra quantum number $n$ for the projectile state. This relates to the incoming wave boundary condition on the coupled channels solution of the core-valence wave function. One needs to include all possible incoming waves for each of the coupled channels scattering states. This extra quantum number carries the information regarding the final state of the core-valence system in the CDCC equations.

\section{STRUCTURE OF ${ }^{11} \mathrm{BE}$ AND ${ }^{17} \mathrm{C}$ IN THE ROTATIONAL MODEL}

We use a rotational model to describe the projectile as a coupled core-valence system. The rotational model for ${ }^{17} \mathrm{C}$ is based on the model used in [54]. We use the same potential parameters as [54], given in Table [ We neglect the spin of the valence particle, because we are interested in the effect of the core spin, and the effect of the valence particle spin on the total cross sections is small. This model produces a ${ }^{17} \mathrm{C}$ ground state spin of $J_{P}^{\pi}=2^{+}$. We use a quadrupole mass deformation of $\beta_{2}=0.55$, as in [56]. Modeling the ${ }^{16} \mathrm{C}$ core with two states $\left\{0^{+}, 2^{+}\right\}$, with an excitation energy of $E_{2^{+}}=1.766$ $\mathrm{MeV}$, and including all possible relative angular momentum up to $l=4$, produces four coupled channels in the ground state: $\left[d \otimes 0^{+}\right],\left[s \otimes 2^{+}\right],\left[d \otimes 2^{+}\right],\left[g \otimes 2^{+}\right]$, with 


\begin{tabular}{|c|c|cccc|}
\hline model & interaction & $V$ & $R_{V}$ & $a_{V}$ & $\beta_{2}$ \\
\hline $\mathrm{CC}$ & ${ }^{16} \mathrm{C}\left\{0^{+}, 2^{+}\right\}+\mathrm{n}$ & 62.0 & 3.2 & 0.65 & 0.55 \\
$\mathrm{SP}$ & ${ }^{16} \mathrm{C}\left\{2^{+}\right\}+\mathrm{n}\{d\}$ & 52.7 & 3.2 & 0.7 & - \\
$\mathrm{SP}$ & ${ }^{16} \mathrm{C}\left\{2^{+}\right\}+\mathrm{n}\{s\}$ & 49.4 & 3.2 & 0.7 & - \\
$\mathrm{SP}$ & ${ }^{16} \mathrm{C}\left\{0^{+}\right\}+\mathrm{n}\{d\}$ & 48.5 & 3.2 & 0.7 & - \\
\hline
\end{tabular}

TABLE I: ${ }^{16} \mathrm{C}+\mathrm{n}$ potential parameters for Eq. (C1).

relative probabilities of $0.175,0.103,0.721,0.001$ respectively. This model predicts the dominant channel to be $\left[d \otimes 2^{+}\right]$, which is consistent with the large spectroscopic factor obtained from shell model calculations (this channel was left out of the model space in the rotational model of 54]). The single particle (SP) central potential parameters are taken from [56], and are adjusted to fit the ground state binding energy for each configuration. ${ }^{16} \mathrm{C}$ also has other bound states around $4 \mathrm{MeV}$ in energy, one of which, the $4^{+}$at $E_{4^{+}}=4.142 \mathrm{MeV}$, could be included within our rotational model. In some of the calculations, we include this core state in the projectile continuum although we keep the projectile's ground state description without the $4^{+}$core state for consistency. Using a rms matter radius of $2.70 \mathrm{fm}[60]$ for the ${ }^{16} \mathrm{C}$ core, the rms radius calculated for the deformed ${ }^{17} \mathrm{C}$ is $2.77 \mathrm{fm}$. The rms radius for the single particle $\left[d \otimes 2^{+}\right]$state is 2.78 $\mathrm{fm}$. The weighted sum for the incoherently summed single particle projectile states, has a rms radius of $2.79 \mathrm{fm}$. The experimental determined rms radus is $3.04 \pm 0.11 \mathrm{fm}$ 61.

For ${ }^{11} \mathrm{Be}$ we base our potential parameters on those of 52]. These were fitted to give the correct binding energies of the ground and first excited state, as well as the $\mathrm{B}(\mathrm{E} 1)$ transition between them, using a parity dependent potential. Since again we neglect the spin of the neutron, and therefore no spin-orbit force, we readjust the potential parameters slightly to fit the binding energies of the bound states. The parameters used in the calculation are given in Table $\llbracket$ with the model CC referring to the coupled rotational model for the projectile, where a deformation $\beta_{2}=0.67$ is taken from [52]. This model produces a ${ }^{11} \mathrm{Be}$ ground state with spin $J_{P}=0^{+}$, with two coupled channels: a s-wave neutron coupled to $0^{+}$core, and a d-wave neutron coupled to a $2^{+}$core. The relative probabilities are 0.883 and 0.117 for the $\left[s \otimes 0^{+}\right]$and $\left[d \otimes 2^{+}\right]$states respectively. The single particle model for the core consists of a parity dependent potential as used in [52], which fits the bound states using a central potential. For the states with a $2^{+}$core the potential depth is adjusted to fit the binding energy plus the energy of the $2^{+}$core. Using a rms matter radius of $2.28 \mathrm{fm}$ 62] for the ${ }^{10} \mathrm{Be}$ core, the rms radius calculated for the deformed ${ }^{10} \mathrm{Be}+n$ is $2.99 \mathrm{fm}$. The rms radius for the single particle $0^{+}$core calculation is $3.08 \mathrm{fm}$. The weighted sum for the incoherently summed single particle projectile states has a radius of $3.01 \mathrm{fm}$. These compare reasonably well to the rms radius for ${ }^{11} \mathrm{Be}$ of $2.90 \pm 0.05 \mathrm{fm}[62$. The radii in our few-body model are a bit small, but since we do not compare to data, and only compare to previous work, we

\begin{tabular}{|c|c|c|cccc|}
\hline model & interaction & parity & $V$ & $R_{V}$ & $a_{V}$ & $\beta_{2}$ \\
\hline $\mathrm{CC}$ & ${ }^{10} \mathrm{Be}\left\{0^{+}, 2^{+}\right\}+\mathrm{n}$ & + & 55.25 & 2.483 & 0.65 & 0.67 \\
$\mathrm{CC}$ & ${ }^{10} \mathrm{Be}\left\{0^{+}, 2^{+}\right\}+\mathrm{n}$ & - & 47.00 & 2.483 & 0.65 & 0.67 \\
$\mathrm{SP}$ & ${ }^{10} \mathrm{Be}\left\{0^{+}\right\}+\mathrm{n}$ & + & 55.50 & 2.736 & 0.67 & - \\
$\mathrm{SP}$ & ${ }^{10} \mathrm{Be}\left\{0^{+}\right\}+\mathrm{n}$ & - & 30.48 & 2.736 & 0.67 & - \\
$\mathrm{SP}$ & ${ }^{10} \mathrm{Be}\left\{2^{+}\right\}+\mathrm{n}$ & + & 75.07 & 2.736 & 0.67 & - \\
$\mathrm{SP}$ & ${ }^{10} \mathrm{Be}\left\{2^{+}\right\}+\mathrm{n}$ & - & 39.95 & 2.736 & 0.67 & - \\
\hline
\end{tabular}

TABLE II: ${ }^{10} \mathrm{Be}+\mathrm{n}$ potential parameters for Eq. (C1).

keep the same potential model for consistency.

\section{NUMERICAL CONSIDERATIONS}

In all the calculations the core-valence integrals were performed out to a maximum radius of $r_{\text {match }}=70 \mathrm{fm}$, with a step length of $h=0.05 \mathrm{fm}$. The momentum integrations for each bin [Eq. (12)] were calculated with 60 steps. The multipole transitions were calculated with all possible $K, Q$ and $\lambda$ transitions, with the transition multipolarity truncated to $\Lambda \leq 4$. Partial waves were included up to $L=5000$ for ${ }^{17} \mathrm{C}$ breakup and up to $L=3000$ for ${ }^{11} \mathrm{Be}$ breakup. The radial wave functions were matched to the asymptotic Coulomb functions at a radius of $R_{\text {asym }}=500 \mathrm{fm}$. The spin of the valence particle was neglected and therefore the channel labels all have $j=l$. The spin of the ${ }^{9} \mathrm{Be}$ target was set to zero as it is a spectator in our reaction model. The channel labels refer to the total spin and parity of the projectile $\left(J_{P}^{\pi}\right)$, the angular momentum of the neutron $(l)$, and the spin of the core fragment $(I)$, in the final state.

The XCDCC model space for the ${ }^{17} \mathrm{C}$ reaction where ${ }^{16} \mathrm{C}$ is modeled with two states $\left\{0^{+}, 2^{+}\right\}$, the XCDCC model space is given in Fig. 2 2 For the ${ }^{17} \mathrm{C}$ reaction where ${ }^{16} \mathrm{C}$ is modeled with three states $\left\{0^{+}, 2^{+}, 4^{+}\right\}$, the XCDCC model space is given in Fig. 3 The XCDCC model space for ${ }^{11} \mathrm{Be}$ breakup is given in Fig. 4 The numbers of bins spanning an energy range for each channel in the final state are given in each box, with the bins at regular intervals in momentum. Summaries of the various sizes for the model space are given in table IIII The first column is the projectile model space: which core states are included in the calculation. The next five columns give the number of permutations for each of the variables; the number of coupled channel states $\left(a J_{P} i n\right)$, the number of projectile states $\left(J_{P} i n\right)$, the number of couplings between coupled channel states $\left(P_{a: a^{\prime}}^{K Q \lambda: \Lambda}\right)$, the number of form factors stored $\left(F_{J_{P} i n: J_{P}^{\prime} i^{\prime} n^{\prime}}^{\Lambda}\right)$, and the number of channels in the CDCC equations $(\alpha)$. The deformed core calculations were performed on a SGI Altix 3700, while the single particle calculations were performed on a cluster of AMD Opteron 250 processors. 


\begin{tabular}{|c|c|c|c|c|c|c|c|c|c|}
\hline Projectile & $a J_{P} i n$ & $J_{P} i n$ & $P_{a: a^{\prime}}^{K Q: \Lambda}$ & $F_{J_{P} i n: J_{P}^{\prime} i^{\prime} n^{\prime}}^{\Lambda}$ & $\alpha$ & CPUtime(s) & NCPUS & walltime(h:m) & $\operatorname{Mem}(\mathrm{GB})$ \\
\hline${ }^{10} \mathrm{Be}\left\{0^{+}\right\}+\mathrm{n}$ & - & 90 & - & 7312 & 258 & 20568 & 11 & $00: 35$ & 0.2 \\
\hline${ }^{10} \mathrm{Be}\left\{0^{+}, 2^{+}\right\}+\mathrm{n}$ & 572 & 179 & 3862133 & 58951 & 542 & 342008 & 8 & $13: 32$ & 1.2 \\
\hline${ }^{16} \mathrm{C}\left\{2^{+}\right\}+\mathrm{n}$ & - & 103 & - & 9788 & 344 & 37900 & 1 & $10: 13$ & 0.2 \\
\hline${ }^{16} \mathrm{C}\left\{0^{+}, 2^{+}\right\}+\mathrm{n}$ & 478 & 144 & 2886779 & 41795 & 473 & 206048 & 15 & $03: 59$ & 1.4 \\
\hline${ }^{16} \mathrm{C}\left\{0^{+}, 2^{+}, 4^{+}\right\}+\mathrm{n}$ & 1269 & 244 & 40036229 & 118450 & 546 & 2991231 & 16 & $56: 01$ & 2.5 \\
\hline
\end{tabular}

TABLE III: Size of calculations for the different model spaces. The first column gives the projectile model, with the core states included. The next 5 columns give the number of permutations for various variables that are required. The last 4 columns give total CPU time, the number of CPUs, walltime, and memory requirements to store the form factors.

\begin{tabular}{|c|c|cccccc|}
\hline type & interaction & $V$ & $r_{V}$ & $a_{V}$ & $W$ & $r_{W}$ & $a_{W}$ \\
\hline central & core + target & 123.0 & 0.75 & 0.8 & 65.0 & 0.78 & 0.8 \\
deformed & core + target & 134.1 & 0.75 & 0.8 & 68.15 & 0.75 & 0.8 \\
central & neutron + target $(\mathrm{E}=62 \mathrm{MeV})$ & 34.54 & 1.17 & 0.75 & 13.4 & 1.26 & 0.58 \\
central & neutron + target $(\mathrm{E}=60 \mathrm{MeV})$ & 33.79 & 1.17 & 0.75 & 12.08 & 1.26 & 0.58 \\
\hline
\end{tabular}

TABLE IV: Fragment-Target optical potential parameters. The radius parameters are to be multiplied by $A_{\text {core }}^{1 / 3}+A_{\text {target }}^{1 / 3}$ for the core-target potential and $A_{\text {target }}^{1 / 3}$ for the neutron-target potential. The core-target parameters are used for both ${ }^{16} \mathrm{C}$ and ${ }^{10} \mathrm{Be}$, and the neutron-target parameters are only adjusted slightly for the different energy of the reaction.

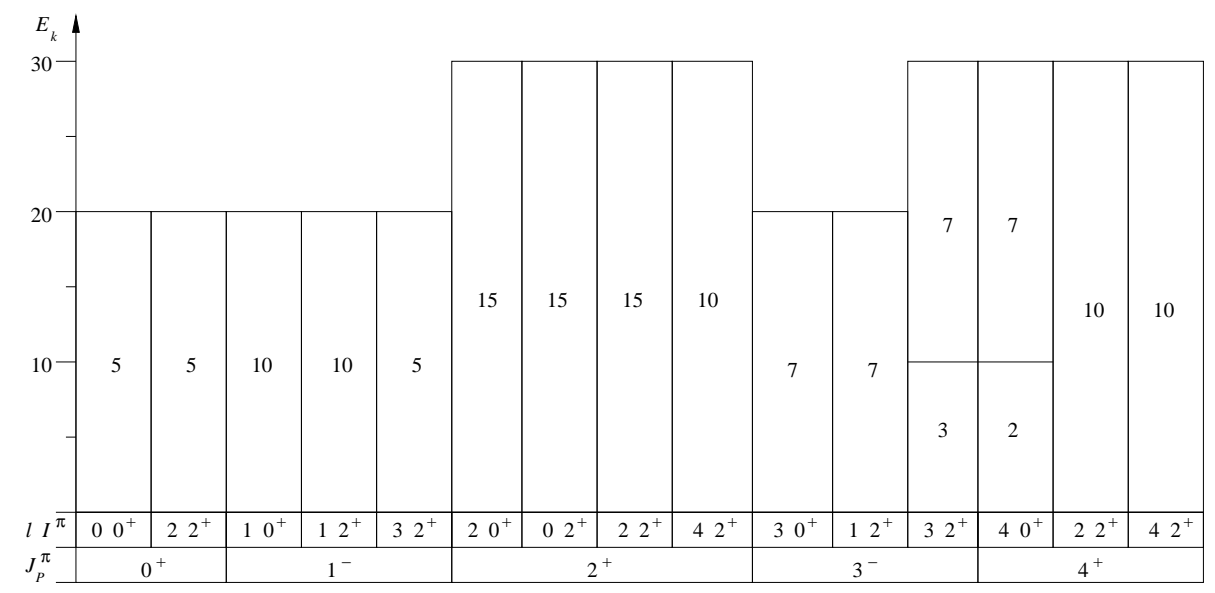

FIG. 2: ${ }^{17} \mathrm{C}$ continuum model space. The number of bins and the energy range are given for each outgoing channel $\left(l, I^{\pi}\right)$ for each spin parity combination of the projectile $\left(J_{P}^{\pi}\right)$.

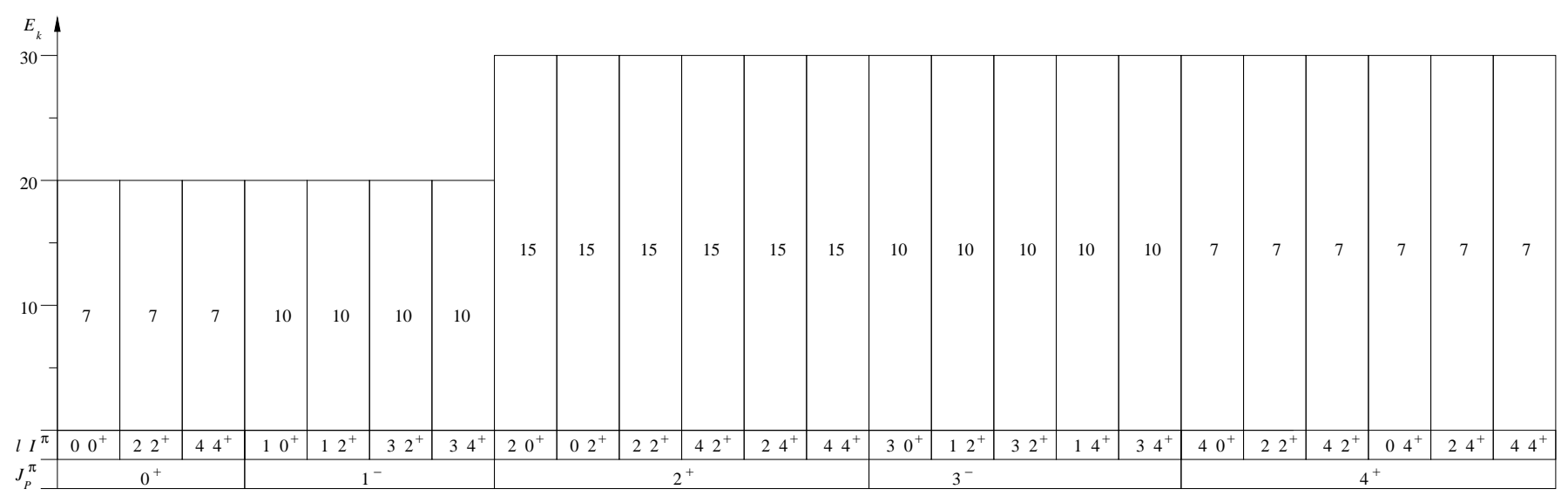

FIG. 3: ${ }^{17} \mathrm{C}$ continuum model space. The number of bins and the energy range are given for each outgoing channel $\left(l, I^{\pi}\right)$ for each spin parity combination of the projectile $\left(J_{P}^{\pi}\right)$. 


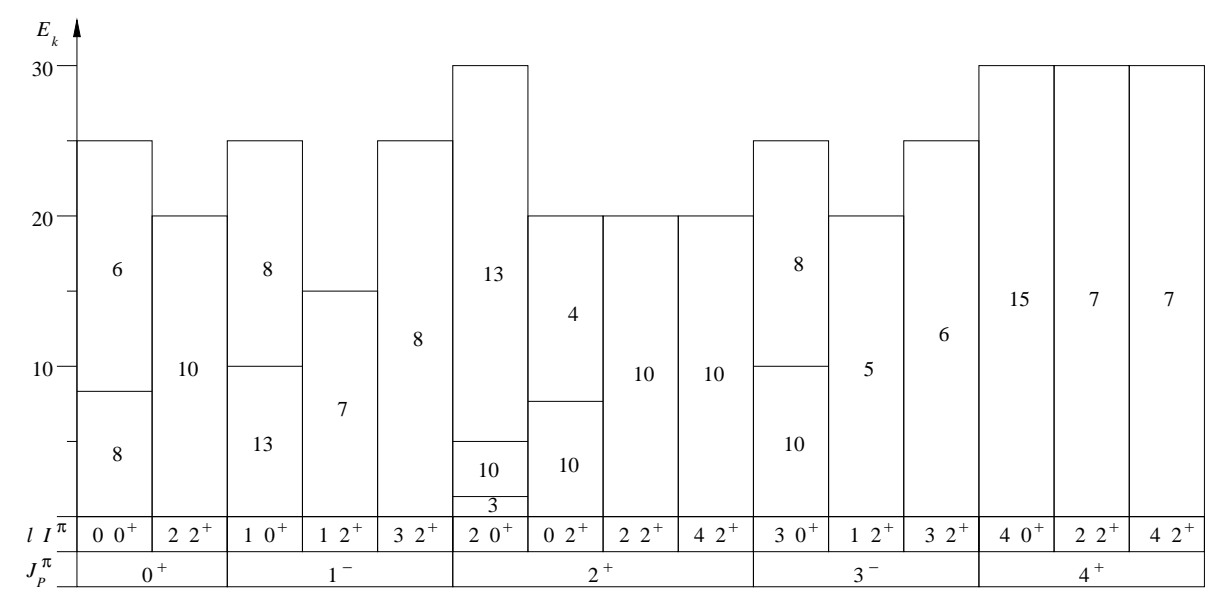

FIG. 4: ${ }^{11}$ Be continuum model space. The number of bins and the energy range are given for each outgoing channel $\left(l, I^{\pi}\right)$ for each spin parity combination of the projectile $\left(J_{P}^{\pi}\right)$.

\section{RESULTS}

Here we present the results of the calculations. For both reactions, we perform three different calculations to understand the relevance of the different ingredients. First we simulate the CDCC version of the single particle calculations in 6, 7]. We refer to it as SPIS: single particle incoherent sum. It corresponds to a sum over the CDCC single particle cross sections multiplied by the corresponding relative probability for the projectile generated in Section IIII It does not contain any interference between core states nor core excitation/de-excitation due to the interaction with the target. Secondly, we perform a calculation where we do not include dynamic effects with the target, and refer to it as CCSE: coupled channels static excitation. It contains the full coupling within the projectile but has only $Q=0$ in the three-body coupling matrix elements. The third case is the full calculation, which we denote by CCDE for coupled channels dynamic excitation. In all the above, we perform the calculations switching on and off continuum-continuum coupling to determine the importance of these couplings as done before [21]. In Tables VIVIII the couplings labelled $\mathrm{c}-\mathrm{v}$ and $\mathrm{c}-\mathrm{T}$ refer to the deformed couplings between the core-valence and core-target subsystems. The monopole couplings are always included.

\section{A. ${ }^{9} \operatorname{Be}\left({ }^{17} \mathrm{C},{ }^{16} \mathrm{C}+n\right) \quad 62 \mathrm{MeV} /$ nucleon}

The 1-step (no continuum-continuum coupling) breakup cross sections for ${ }^{9} \mathrm{Be}\left({ }^{17} \mathrm{C},{ }^{16} \mathrm{C}\left\{0^{+}, 2^{+}\right\}+\mathrm{n}\right)$ are given in Table $\nabla$ The single particle cross sections for each of the configurations used in the $\mathrm{CC}$ calculations are $33 \mathrm{mb}, 25 \mathrm{mb}$ and $13 \mathrm{mb}$ for the $\left[d \otimes 0^{+}\right],\left[s \otimes 2^{+}\right]$, and $\left[d \otimes 2^{+}\right]$states respectively (we neglect the $\left[g \otimes 2^{+}\right]$ state since its contribution is very small). These are then multiplied by $0.175,0.103$, and 0.721 to obtain the single particle incoherent sum SPIS cross section of $18 \mathrm{mb}$. This

\begin{tabular}{|c||c|c||c|c||c|}
\hline \multirow{2}{*}{\multicolumn{1}{|c||}{ Model }} & \multicolumn{2}{c||}{ Couplings } & \multicolumn{1}{|c||}{} & \\
\cline { 2 - 5 } & $\mathrm{c}-\mathrm{v}$ & $\mathrm{c}-\mathrm{T}$ & $\sigma_{0+}$ & $\sigma_{2+}$ & $\sigma$ \\
\hline SPIS & $\times$ & $\times$ & 6 & 12 & 18 \\
CCSE & $\checkmark$ & $\times$ & 5 & 11 & 17 \\
CCDE & $\checkmark$ & $\checkmark$ & 7 & 14 & 21 \\
\hline
\end{tabular}

TABLE V: ${ }^{17} \mathrm{C}$ 1-step (no CC couplings) breakup cross sections leading to ${ }^{16} \mathrm{C}\left\{0^{+}, 2^{+}\right\}+\mathrm{n}$ : SPIS is the single particle incoherent sum model, where neither core-valence or coretarget excitation couplings are included, CCSE is the coupled channel static excitation calculations, where core-valence couplings are included but no core-target, and finally the full calculation CCDE coupled channel dynamic excitation, where both core-valence or core-target excitation couplings are included.

model is equivalent to that used widely to analyze knockout data to specific states [47], the difference being that this is a 1-step breakup reaction model which includes nuclear and Coulomb breakup whereas the eikonal model includes single-particle-continuum-continuum couplings but nuclear breakup only. We will include continuumcontinuum couplings in our model later. Introducing a coupled channels description of the projectile (CCSE) provides very similar cross sections when comparing to the SPIS model. This suggests little interference between configurations during the reaction. Including dynamical excitation in the reaction model (CCDE) enhances the cross section to both core states, increasing the total breakup cross section to $21 \mathrm{mb}$ in agreement with the findings of Batham et al. [56]. However in that work, only cross sections summed over all core states were given.

We explore the relevance of other states in ${ }^{16} \mathrm{C}$ by first calculating the inelastic reaction ${ }^{9} \mathrm{Be}\left({ }^{16} \mathrm{C}\left(2^{+}\right),{ }^{16} \mathrm{C}^{*}\right)$. We use a simple rotational model for ${ }^{16} \mathrm{C}$, and include three states $\left\{0^{+}, 2^{+}, 4^{+}\right\}$. We have ${ }^{16} \mathrm{C}\left(2^{+}\right)$in the initial state since ${ }^{17} \mathrm{C}$ has predominantly ${ }^{16} \mathrm{C}\left(2^{+}\right)$in the ground state. The resulting inelastic cross section to specific core states 


\begin{tabular}{|c||c|c|c||c|c|c||c|}
\hline \multirow{2}{*}{ Model } & \multicolumn{3}{|c|}{ Couplings } & & & & \\
\cline { 2 - 7 } & $\mathrm{C}-\mathrm{v}$ & $\mathrm{c}-\mathrm{T}$ & $\mathrm{CC}$ & $\sigma_{0+}$ & $\sigma_{2+}$ & $\sigma_{4+}$ & $\sigma$ \\
\hline SPIS & $\times$ & $\times$ & $\times$ & 6 & 12 & - & 18 \\
CCSE & $\checkmark$ & $\times$ & $\times$ & 6 & 12 & 5 & 22 \\
CCDE & $\checkmark$ & $\checkmark$ & $\times$ & 7 & 15 & 5 & 27 \\
\hline SPIS & $\times$ & $\times$ & $\checkmark$ & 5 & 11 & - & 16 \\
CCDE & $\checkmark$ & $\checkmark$ & $\checkmark$ & 4 & 10 & 5 & 19 \\
\hline
\end{tabular}

TABLE VI: ${ }^{17} \mathrm{C}$ breakup cross sections leading to ${ }^{16} \mathrm{C}\left\{0^{+}, 2^{+}, 4^{+}\right\}+\mathrm{n}$. The abreviations in the first row are the same as in Table $\nabla$ Calculations are repeated with and without continuum-continuum couplings (labeled here by $\mathrm{CC}$ ).

is $\sigma_{0^{+}}=4 \mathrm{mb}, \sigma_{2^{+}}=5 \mathrm{mb}$, and $\sigma_{4^{+}}=12 \mathrm{mb}$. The large number of projections of the $4^{+}$state and its relatively low energy $E_{4^{+}}=4.142 \mathrm{Mev}$, raises suspicion that this $4^{+}$state will hold a significant contribution to the breakup cross section of ${ }^{17} \mathrm{C}$. We recognize that the $2^{+}$ excited state in ${ }^{16} \mathrm{C}$ is not described well in a collective model, but here we use it as a toy model to compare with previous work. One may also wonder about contributions from other states in a rotational band, since we are using the particle-rotor model. We include a $4^{+}$state here since we start with ${ }^{16} \mathrm{C}$ in a $2^{+}$state. Inelastic excitations with $\Delta I=4$, within our rotational model, are an order of magnitude smaller than the $\Delta I=2$ transitions, thus it should only be necessary to include the $\Delta I=2$ states for the core in our rotational model.

We therefore recalculate the cross section for ${ }^{17} \mathrm{C}$ breakup including the $4^{+}$state in the continuum (Table VI). We keep the same ground state with only $\left\{0^{+}, 2^{+}\right\}$so that the relative weights of each ground state configuration are preserved. Since we have no $4^{+}$in the ground state, the SPIS model is the same as before. The $4^{+}$state enhances the total breakup cross section in the CCSE model from $17 \mathrm{mb}$ to $22 \mathrm{mb}$ and in the CCDE model from 21 to $27 \mathrm{mb}$. The last two rows in Table V1] are calculations including continuum-continuum couplings. These couplings suppress the cross section as was seen before in the single particle case 19, 41]. It is a mere coincidence, though, that the final total cross section of $19 \mathrm{mb}$ compares so well with the SPIS result.

\section{B. ${ }^{9} \mathrm{Be}\left({ }^{11} \mathrm{Be},{ }^{11} \mathrm{Be}+n\right) \quad 60 \mathrm{MeV} /$ nucleon}

The breakup cross sections for the reaction ${ }^{9} \mathrm{Be}\left({ }^{11} \mathrm{Be},{ }^{10} \operatorname{Be}\left\{0^{+}, 2^{+}\right\}+\mathrm{n}\right)$ are shown in Table VII The single particle cross sections for 1-step breakup are $159 \mathrm{mb}$ for the $\left[s \otimes 0^{+}\right]$state, and $10 \mathrm{mb}$ for the $\left[d \otimes 2^{+}\right]$ state. Multiplying these single particle cross sections by the relative probabilities of 0.883 and 0.117 respectively, one gets a total breakup cross section of $141 \mathrm{mb}$ for the SPIS model. If we calculate the cross section in the CCSE, the cross section drops to $135 \mathrm{mb}$, suggesting some destructive interference between the configurations. When we include dynamical excitation (CCDE) the cross section is enhanced at $150 \mathrm{mb}$. The apparent

\begin{tabular}{|c||c|c|c||c|c||c|}
\hline \multirow{2}{*}{ Model } & \multicolumn{3}{c|}{ Couplings } & & \multirow{1}{*}{} & \\
\cline { 2 - 5 } C-v & c-T & CC & $\sigma_{0+}$ & $\sigma_{2+}$ & $\sigma$ \\
\hline SPIS & $\times$ & $\times$ & $\times$ & 140 & 1 & 141 \\
CCSE & $\checkmark$ & $\times$ & $\times$ & 128 & 7 & 135 \\
CCDE & $\checkmark$ & $\checkmark$ & $\times$ & 142 & 8 & 150 \\
\hline SPIS & $\times$ & $\times$ & $\checkmark$ & 109 & 1 & 110 \\
CCSE & $\checkmark$ & $\times$ & $\checkmark$ & 107 & 8 & 115 \\
CCDE & $\checkmark$ & $\checkmark$ & $\checkmark$ & 109 & 8 & 117 \\
\hline
\end{tabular}

TABLE VII: ${ }^{11}$ Be nuclear and Coulomb breakup cross sections for ${ }^{10} \mathrm{Be}\left\{0^{+}, 2^{+}\right\}+\mathrm{n}$ The abreviations in the first row are the same as in Table $\mathrm{V}$

agreement between the cross section to the $0^{+}$state $(142$ $\mathrm{mb}$ ) in the CCDE model and the single particle cross section multiplied by the relative probability $(140 \mathrm{mb})$ is merely a coincidence. It is a result of cancellations between two different effects, the interference between different configurations of ${ }^{11} \mathrm{Be}$ and the dynamical excitation couplings in the core-target potential. We also see that the SPIS model severely underpredicts the amount of cross section to the $2^{+}$state. The additional cross section seems to come from including the couplings between the ${ }^{10} \mathrm{Be}$ and the neutron rather than from the dynamical excitation couplings of the core-target interaction. When continuum-continuum couplings are included there is a general reduction of the cross sections, but the same qualitative effects of projectile couplings and dynamical excitation remain.

In the eikonal model of breakup reactions used in the analysis of knockout data 47], only nuclear breakup is included. In order to compare with previous calculations for this reaction 7], where the contributions from Coulomb breakup and dynamical excitation were roughly estimated, we rerun our calculations with only nuclear breakup. Result for the CCDE show that the nuclear cross section to the $0^{+}$state is $10 \mathrm{mb}$ less than the cross section calculated when both nuclear and Coulomb are included. This appears to agree well with the estimate of $10 \mathrm{mb}$ given in [7], however it does not mean that Coulomb and nuclear should be added incoherently. A Coulomb breakup only calculation yields $12 \mathrm{mb}$. The estimated contribution from dynamical excitation of $0^{+}$ core to the $2^{+}$excited state was $11 \mathrm{mb}$ multiplied by the spectroscopic factor of the $0^{+}$core, equivalent to 0.883 in our rotational model, which gives $10 \mathrm{mb}$. We see from Table VIII that the increase in the cross section to the $2^{+}$state, from the SPIS to our CCDE model is $7 \mathrm{mb}$. So it turns out that the estimates presented in [7] produced total cross sections close to those we obtain in a more accurate calculation. Again, it is important to note that in general this will not happen. A comparison of the knockout data from [7] within this new model which incorporates core deformation in a consistent manner is given in 46]. 


\begin{tabular}{|c||c|c|c||c|c||c|}
\hline \multirow{2}{*}{ Model } & \multicolumn{3}{|c|}{ Couplings } & & \multirow{1}{*}{} & \\
\cline { 2 - 5 } & $\mathrm{C}-\mathrm{v}$ & $\mathrm{c}-\mathrm{T}$ & $\mathrm{CC}$ & $\sigma_{0+}$ & $\sigma_{2+}$ & $\sigma$ \\
\hline SPIS & $\times$ & $\times$ & $\checkmark$ & 103 & 1 & 104 \\
CCDE & $\checkmark$ & $\checkmark$ & $\checkmark$ & 100 & 7 & 107 \\
\hline
\end{tabular}

TABLE VIII: ${ }^{11}$ Be nuclear breakup cross sections for ${ }^{10} \operatorname{Be}\left\{0^{+}, 2^{+}\right\}+\mathrm{n}$. The abreviations in the first row are the same as in Table $\nabla$

\section{SUMMARY AND CONCLUSIONS}

In summary, we have extended the CDCC method to include a coupled channels description of the projectile, using a deformed core-valence interaction, thus allowing excited core contributions in the projectile states. We also include a deformed core-target interaction, allowing the core to (de-)excite during the reaction.

For the reactions considered here, the breakup of ${ }^{17} \mathrm{C}$ and ${ }^{11} \mathrm{Be}$ on a ${ }^{9} \mathrm{Be}$ target at $\approx 60 \mathrm{MeV} /$ nucleon, the comparison of the structure calculations with the experimental data will depend on the reaction model used. By including more of the important dynamics of the reaction mechanisms, a more direct comparison of structure and experiment will be possible. Even though the excited states of ${ }^{16} \mathrm{C}$ are not well described by collective excitations, we use a toy model of rotational excitations to the excited $2^{+}$and $4^{+}$states. This is to compare with previous calculations. In the breakup of ${ }^{17} \mathrm{C}$ we find that total cross section is significantly increased due to excitation of the core, which is consistent with previous calculations. Our calculations here go beyond previous work allowing for a partial wave decomposition of the final core states. Even when there is no $4^{+}$core-state in the ground state of ${ }^{17} \mathrm{C}$, significant population of the $4^{+}$is predicted in the final breakup states. Continuumcontinuum couplings decrease the cross section.

The particle-rotor model for ${ }^{11} \mathrm{Be}$ describes the nucleus well, and there is good agreement with the spectroscopic factors obtained from shell model calculations. When we allow the ${ }^{10}$ Be to deform, we see an increase in the total breakup cross section compared to the weighted incoherent sum of the single particle cross sections. The cross section to the $2^{+}$state in ${ }^{10} \mathrm{Be}$ is significantly increased when the core is deformed.

From our calculations we conclude that both the deformed couplings between the core and valence particles and the deformed couplings between the core and target are important and should be included in the reaction model. This eXtended CDCC (XCDCC) reaction model can now be used to describe breakup reactions where significant contributions from excited core states are needed to describe the projectile at any point during the reaction process. The usefulness of XCDCC is varied. Near future applications of XCDCC include ${ }^{11} \mathrm{Be}\left(\mathrm{p}, \mathrm{p}^{\prime}\right)^{11} \mathrm{Be}^{*}$, ${ }^{12} \mathrm{C}\left({ }^{11} \mathrm{Be},{ }^{10} \mathrm{Be}+\mathrm{n}\right)$, and the various modes of ${ }^{8} \mathrm{~B}$ breakup. A comparison with breakup data to specific core states will allow accurate checks of the validity of the structure model for the projectile and thereby improve the compar- ison between structure calculations and experiment. We will also be able to look at detailed breakup spectra and analyze resonances built on excited core states, which could not be studied using previous reaction models.

The generality of the formalism for including core degrees of freedom in XCDCC shows that one can go beyond the simple particle-rotor model for the projectile. Given the radial overlap wave functions between the projectile and the core states, and the core elastic and inelastic transitions for the core-target scattering, a more microscopic picture of the reaction can be obtained, including important dynamical effects which can have a significant effect on the reaction observables.

\section{Acknowledgements}

We thank Jeff Tostevin for useful discussions. We thank the high performance computing center (HPCC) at MSU for the use of their facilities. This work is supported by NSCL, Michigan State University, the U.K. EPSRC through grant GR/T28577, and the National Science Foundation through grant PHY-0456656.

\section{APPENDIX A: DETAILS ON THE THREE-BODY COUPLING POTENTIALS}

Here we give the details of the derivation for Eq. (23). The expansion in $Q$, Eq. (18), and $\lambda$, Eq. (20), gives us three spherical harmonics which depend on the $\hat{\boldsymbol{R}}, \hat{\boldsymbol{r}}$ and $\xi$ degrees of freedom. The expansion in $K$, Eq. (21), gives us two spherical harmonics in $\hat{\boldsymbol{R}}$ and $\hat{\boldsymbol{r}}$, which combine with the spherical harmonics from the $Q$ expansion to give two new multipole orders, $\Lambda$ and $\Lambda^{\prime}$, which relate to the projectile-target and core-valence relative motion respectively:

$$
\begin{aligned}
& C_{K k}(\hat{\boldsymbol{R}}) C_{\lambda \mu}(\hat{\boldsymbol{R}})= \sum_{\Lambda \omega} C_{\Lambda \omega}(\hat{\boldsymbol{R}}) \hat{\Lambda}^{2}(-1)^{\omega} \\
& \times\left(\begin{array}{ccc}
K & \lambda & \Lambda \\
k & \mu & -\omega
\end{array}\right)\left(\begin{array}{ccc}
K & \lambda & \Lambda \\
0 & 0 & 0
\end{array}\right), \\
& C_{K-k}(\hat{\boldsymbol{r}}) C_{Q-\lambda, q-\mu}(\hat{\boldsymbol{r}})= \sum_{\Lambda^{\prime} \omega^{\prime}} C_{\Lambda^{\prime} \omega^{\prime}}(\hat{\boldsymbol{r}}) \hat{\Lambda}^{\prime 2}(-1)^{\omega^{\prime}} \\
& \times\left(\begin{array}{ccc}
K & Q-\lambda & \Lambda^{\prime} \\
-k & q-\mu & -\omega^{\prime}
\end{array}\right)\left(\begin{array}{ccc}
K & Q-\lambda & \Lambda^{\prime} \\
0 & 0 & 0
\end{array}\right) .
\end{aligned}
$$

We then combine the three spherical harmonics, for the three coordinates, to form a tripolar spherical harmonic with zero total angular momentum, which is a tensor of rank zero, since the matrix elements of the potential 
operator must conserve total angular momentum:

$$
\begin{array}{r}
C_{\Lambda \omega}(\hat{\boldsymbol{R}}) C_{\Lambda^{\prime} \omega^{\prime}}(\hat{\boldsymbol{r}}) C_{Q-q}(\xi)=\frac{(-1)^{\Lambda^{\prime}-Q}}{\hat{\Lambda}}\left(\begin{array}{ccc}
\Lambda & \Lambda^{\prime} & Q \\
\omega & \omega^{\prime} & -q
\end{array}\right) \\
\times\left(C_{\Lambda}(\hat{\boldsymbol{R}}) \cdot\left\{C_{\Lambda^{\prime}}(\hat{\boldsymbol{r}}) \otimes C_{Q}(\xi)\right\}_{\Lambda}\right) .
\end{array}
$$

Using these definitions, one can write the potential oper- ator as Eq. (23).

In order to calculate the matrix elements, we use the projectile coupling order defined in Eqs. (3) \& (6). The matrix elements of the tripolar spherical harmonic of rank zero is:

$$
\begin{aligned}
& \left\langle\left(L\left[(l s) j, I ; J_{P}\right]\right) J, J_{t} ; J_{T}\left|\left(C_{\Lambda}(\hat{\boldsymbol{R}}) \cdot\left\{C_{\Lambda^{\prime}}(\hat{\boldsymbol{r}}) \otimes C_{Q}(\xi)\right\}_{\Lambda}\right)\right|\left(L^{\prime}\left[\left(l^{\prime} s\right) j^{\prime}, I^{\prime} ; J_{P}^{\prime}\right]\right) J^{\prime}, J_{t}^{\prime} ; J_{T}\right\rangle \\
& =\delta_{J_{t}, J_{t}^{\prime}} \delta_{J, J^{\prime}} \delta_{s, s^{\prime}}(-1)^{J_{P}+J+\Lambda+j^{\prime}+l+l^{\prime}+s} \hat{J}_{P} \hat{J}_{P}^{\prime} \hat{L} \hat{L}^{\prime} \hat{j} \hat{j}^{\prime} \hat{l} \hat{l}^{\prime} \hat{\Lambda} \\
& \times\left(\begin{array}{ccc}
\Lambda & L & L^{\prime} \\
0 & 0 & 0
\end{array}\right)\left(\begin{array}{ccc}
\Lambda^{\prime} & l & l^{\prime} \\
0 & 0 & 0
\end{array}\right)\left\{\begin{array}{ccc}
J_{P} & J_{P}^{\prime} & \Lambda \\
L^{\prime} & L & J
\end{array}\right\}\left\{\begin{array}{ccc}
j & j^{\prime} & \Lambda^{\prime} \\
l^{\prime} & l & s
\end{array}\right\}\left\{\begin{array}{cccc}
J_{P} & J_{P}^{\prime} & \Lambda \\
j & j^{\prime} & \Lambda^{\prime} \\
I & I^{\prime} & Q
\end{array}\right\}\left\langle I\left\|C_{Q}(\xi)\right\| I^{\prime}\right\rangle .
\end{aligned}
$$

By rearranging the summations, one can write the transition potentials as defined in Eqs. (24) 27).

The reduced matrix element for the core degree of freedom is defined using the Bohr \& Mottelson definition:

$$
\left\langle I\left\|C_{Q}(\xi)\right\| I^{\prime}\right\rangle=\hat{I}\left\langle I\left|C_{Q}(\xi)\right| I^{\prime}\right\rangle /\left(I^{\prime} K_{c}^{\prime} Q q \mid I K_{c}\right) .
$$

We have left this matrix element separate since the derivation of the coupling potential is independent of the choice for the core inelastic excitation mode. The specific form for the matrix element within a rotational model is presented in Appendix C.

\section{APPENDIX B: $Q=0$ LIMIT}

If one sets $Q=0$ in the transition potential defined in Eqs. (24 27), this corresponds to the limit that the core cannot dynamically excite during the reaction, hence the core spin in the initial state is the same as the core spin in the final state. In the $Q=0$ limit, the coupling potentials are the same as for single particle breakup as defined in [58], since $\Lambda=K$,

$$
\begin{aligned}
U_{\alpha: \alpha^{\prime}}^{J_{T}}(R)= & \hat{L} \hat{L}^{\prime} \hat{J}_{P} \hat{J}_{P}^{\prime}(-1)^{J_{P}+J} \sum_{K}(-1)^{K} \hat{K}^{2} \\
& \times\left(\begin{array}{ccc}
K & L & L^{\prime} \\
0 & 0 & 0
\end{array}\right)\left\{\begin{array}{ccc}
J_{P} & J_{P}^{\prime} & K \\
L^{\prime} & L & J
\end{array}\right\} F_{J_{P} i n: J_{P}^{\prime} i^{\prime} n^{\prime}}^{K}(R),
\end{aligned}
$$

where the form-factors are the sum over the coupled channels states of the radial integrals and couplings,

$$
F_{J_{P} i n: J_{P}^{\prime} i^{\prime} n^{\prime}}^{K}(R)=\sum_{a a^{\prime}} R_{a i n: a^{\prime} i^{\prime} n^{\prime}}^{K 00}(R) P_{a: a^{\prime}}^{K 00: K} .
$$

The radial integrals are

$$
R_{a i n: a^{\prime} i^{\prime} n^{\prime}}^{K 00}(R)=\hat{K} \int_{0}^{R_{m}} u_{a: n}^{i *}(r) V_{c t}^{0 K}(r, R) u_{a^{\prime}: n^{\prime}}^{i^{\prime}}(r) \mathrm{d} r
$$

and the couplings are

$$
\begin{aligned}
P_{a: a^{\prime}}^{K 00: K}= & \delta_{I I^{\prime}}(-1)^{j^{\prime}+l+l^{\prime}+s+J_{P}^{\prime}+j+I} \hat{K} \hat{j} \hat{j^{\prime}} \hat{\prime} \hat{l^{\prime}} \\
& \times\left(\begin{array}{ccc}
K & l & l^{\prime} \\
0 & 0 & 0
\end{array}\right)\left\{\begin{array}{ccc}
J_{P} & J_{P}^{\prime} & K \\
j^{\prime} & j & I
\end{array}\right\}\left\{\begin{array}{ccc}
j & j^{\prime} & K \\
l^{\prime} & l & s
\end{array}\right\} .
\end{aligned}
$$

One can see from this limit that once the form-factors are calculated, the coupling potentials have same form as with core excitation. We associate this limit with a reaction model where the core cannot dynamically excite due to the interaction with the target. This limit may still include couplings between the core and valence particles which can excite/de-excite the core.

\section{APPENDIX C: ROTATIONAL MODEL FOR THE CORE}

In the formalism for XCDCC, we did not assume a model for the core-valence coupling. Here we evaluate the XCDCC equations using a rotational model for the core. For the radial dependence, we use a deformed WoodsSaxon potential

$$
V_{v c}(\boldsymbol{r}, \xi)=\frac{V}{1+\exp \left[(r-R(\xi)) / a_{V}\right]},
$$

where the radius depends on the quadrupole deformation:

$$
R(\xi)=R_{V}\left(1+\beta_{2} Y_{20}(\xi)\right) .
$$


The core-valence potential are numerically calculated by expanding in multipoles, as defined in [58],

$$
V_{v c}(\boldsymbol{r}, \xi)=\sum_{Q} V_{v c}^{Q}(\boldsymbol{r}) P_{Q}(\xi)
$$

In the rotational model, the eigenstates of the core, $\varphi_{I}(\xi)$, are proportional to the rotational matrices,

$$
\langle\xi \mid I\rangle=\frac{I}{\sqrt{8 \pi^{2}}} \mathcal{D}_{K_{c} 0}^{I}(\xi) .
$$

The matrix element, for the core degrees of freedom, in the coupling potentials is then

$$
\left\langle I\left\|C_{Q}(\xi)\right\| I^{\prime}\right\rangle=(-1)^{I^{\prime}+K_{c}} \hat{I} \hat{I}^{\prime}\left(\begin{array}{ccc}
Q & I & I^{\prime} \\
0 & -K_{c} & K_{c}
\end{array}\right),
$$

where $K_{c}$ is the projection of the core spin, $I$, on the body-fixed z-axis.
[1] B. Jonson, Phys. Rep. 389, 1 (2004).

[2] J. S. Al-Khalili and F. M. Nunes, J. Phys. G 29, R89 (2003).

[3] T. Nakamura et al., Phys. Rev. Lett. 83, 001112 (1999).

[4] J. H. Kelley et al., Phys. Rev. Lett. 74, 30 (1995).

[5] N. A. Orr et al., Phys. Rev. C 51, 3116 (1995).

[6] V. Maddalena et al., Phys. Rev. C 63, 024613 (2001).

[7] T. Aumann et al., Phys. Rev. Lett. 84, 35 (2000).

[8] V. Maddalena and R. Shyam, Phys. Rev. C 63, 051601(R) (2001).

[9] N. Fukuda et al., Phys. Rev. C 70, 054606 (2004).

[10] B. M. Young et al., Phys. Rev. C 49, 279 (1994).

[11] K. Alder and A. Winther, Electromagnetic Excitation (North-Holland, Amsterdam, 1975).

[12] G. Baur, K. Hencken, and D. Trautmann, Prog. Part. Nucl. Phys. 51, 487 (2003).

[13] T. Motobayashi et al., Phys. Lett. B264, 259 (1991).

[14] T. Kikuchi et al., Phys. Lett. B391, 261 (1997).

[15] B. Davids et al., Phys. Rev. Lett. 81, 2209 (1998).

[16] F. Fleurot et al., Phys. Lett. B615, 167 (2005).

[17] H. Esbensen and G. F. Bertsch, Nucl. Phys. A600, 37 (1996).

[18] C. H. Dasso, S. M. Lenzi, and A. Vitturi, Nucl. Phys. A611, 124 (1996); A639, 635 (1998).

[19] F. M. Nunes and I. J. Thompson, Phys. Rev. C 57, R2818 (1998).

[20] H. Esbensen and G. F. Bertsch, Phys. Rev. C 59, 3240 (1999).

[21] F. M. Nunes and I. J. Thompson, Phys. Rev. C 59, 2652 (1999).

[22] P. Capel and D. Baye, Phys. Rev. C 71, 044609 (2005).

[23] K. Ogata, S. Hashimoto, Y. Iseri, M. Kamimura, and M. Yahiro, arvix.org/nucl-th/0505007 (2005), submitted to Phys. Rev. C.

[24] H. Esbensen, G. F. Bertsch, and K. A. Snover, Phys. Rev. Lett. 94, 042502 (2005).

[25] J. J. Kolata et al., Phys. Rev. C 63, 024616 (2001).

[26] D. Cortina-Gil et al., Nucl. Phys. A720, 69 (2003).

[27] F. M. Marques et al., Phys. Lett. B476, 219 (2000).

[28] J. A. Tostevin, S. Rugmai and R. C. Johnson, Phys. Rev. C 57, 3225 (1998).

[29] E. Garrido, D. V. Fedorov and A. S. Jensen, Phys. Rev. C 59, 1272 (1999).

[30] Y. Sakuragi, M. Yahiro, and M. Kamimura, Prog. Theor. Phys. Suppl. 89, 136 (1986).

[31] J. A. Tostevin, F. M. Nunes, and I. J. Thompson, Phys. Rev. C 63, 024617 (2001).

[32] T. Matsumoto, E. Hiyama, K. Ogata, Y. Iseri, M.
Kamimura, S. Chiba, and M. Yahiro, Phys. Rev. C 70, 061601(R) (2004).

[33] T. Egami, K. Ogata, T. Matsumoto, Y. Iseri, M. Kamimura, and M. Yahiro, Phys. Rev. C 70, 047604 (2004).

[34] M. Kamimura, T. Matsumoto, E. Hiyama, K. Ogata, Y. Iseri, and M. Yahiro, AIP Conf. Proc. 791, 174 (2005).

[35] T. Matsumoto, T. Egami, K. Ogata, Y. Iseri, M. Kamimura, and M. Yahiro, arvix.org/nucl-th/0511059 (2005), submitted to Phys. Rev. C.

[36] C. A. Bertulani, Phys. Rev. Lett. 94, 072701 (2005).

[37] E. O. Alt, B. F. Irgaziev, and A. M. Mukhamedzhanov, Phys. Rev. C 71, 024605 (2005).

[38] K. Rusek and K. W. Kemper, Phys. Rev. C 61, 034608 (2000).

[39] M. Takashina, S. Takagi, Y. Sakuragi, and Y. Iseri, Phys. Rev. C 67, 037601 (2003).

[40] A. M. Moro, R. Crespo, F. M. Nunes, and I. J. Thompson, Phys. Rev. C 67, 047602 (2003).

[41] N. C. Summers and F. M. Nunes, Phys. Rev. C 70, 011602(R) (2004).

[42] B. Davids, Sam M. Austin, D. Bazin, H. Esbensen, B. M. Sherrill, I. J. Thompson, and J. A. Tostevin, Phys. Rev. C 63, 065806 (2001).

[43] J. Mortimer, I. J. Thompson, and J. A. Tostevin, Phys. Rev. C 65, 064619 (2002).

[44] N. C. Summers and F. M. Nunes, J. Phys. G 31, 1437 (2005).

[45] A. Shrivastava et al., Phys. Lett. B596, 54 (2004).

[46] N. C. Summers, F. M. Nunes, and I. J. Thompson, Phys. Rev. C (R) (to be published).

[47] P. G. Hansen and J. A. Tostevin, Annu. Rev. Nucl. Part. Sci. 53, 219 (2003).

[48] J. A. Tostevin, J. Phys. G 23, 735 (1999).

[49] J. A. Tostevin, Nucl. Phys. A682, 320c (2001).

[50] J. A. Tostevin, D. Bazin, B. A. Brown, T. Glasmacher, P. G. Hansen, V. Maddalena, A. Navin, and B. M. Sherrill, Phys. Rev. C 66, 024607 (2002).

[51] R. Shyam and P. Danielewicz, Phys. Rev. C 63, 054608 (2001).

[52] F. M. Nunes, I. J. Thompson, and R. C. Johnson, Nucl. Phys. A596, 171 (1996).

[53] F. M. Nunes, J. A. Christley, I. J. Thompson, R. C. Johnson, and V. D. Efros, Nucl. Phys. A609, 43 (1996).

[54] D. Ridika, M. H. Smedberg, J. S. Vaagen, and M. V. Zhukov, Nucl. Phys. A628, 363 (1998).

[55] T. Tarutina and M. S. Hussein, Phys. Rev. C 70, 034603 (2004). 
[56] P. Batham, I. J. Thompson, and J. A. Tostevin, Phys. Rev. C 71, 064608 (2005).

[57] J. A. Christley and I. J. Thompson, Comp. Phys. Comm. 79, 143 (1994).

[58] I. J. Thompson, Comput. Phys. Rep. 7, 3 (1988).

[59] M. Moshinsky, Nucl. Phys. 13, 104 (1959).
[60] A. Ozawa et al., Nucl. Phys. A693, 32 (2001).

[61] M. G. Saint-Laurent et al., Z. Phys. A 332, 457 (1989).

[62] J. S. Al-Khalili, J. A. Tostevin, and I. J. Thompson, Phys. Rev. C 54, 1843 (1996). 